\title{
Resource Allocation in Spectrum Deployment for Cognitive Third-party Users
}

\author{
Arikatla Jaya Lakshmi ${ }^{1}$ \\ Research Scholar, Department of \\ ECE, Jawaharlal Nehru \\ Technological University, Anantapur \\ Ananthapuramu, Andhra Pradesh, \\ India
}

\author{
Dr.G.N.Swamy ${ }^{2}$ \\ Professor and HOD, Department of \\ EIE, V R Siddhartha Engineering \\ College, Vijayawada \\ Andhra Pradesh \\ India
}

\author{
Dr.M.N.Giri Prasad ${ }^{3}$ \\ Professor, Department of ECE \\ Jawaharlal Nehru Technological \\ University, Anantapur \\ Ananthapuramu, Andhra Pradesh, \\ India
}

\begin{abstract}
Spectrum scarcity is a major challenge in wireless communication for next generation applications. The spectrum sharing and utilization of other user available spectrum is an optimal solution, which has outcome with a new mode of communication called cognitive network. Cognitive devices are capable of requesting and sharing free spectrum among each other in a communication range. The spectrums are shared among primary and secondary user (PU, SU). To extend the range of spectrum utilization, users from other clusters are requested in sharing of spectrum which is called as third party user (TSU). In detection of free spectrum a jamming based approach is proposed in recent work. Jamming approach generates a periodic jamming signal for TSU in sensing of free spectrum. The repetitive requesting of spectrum availability result in large jamming probability for engaged TSU users resulting in large delay. In minimizing the delay observed, in this paper, a new monitored jamming approach is proposed. Proposed Monitored jamming approach is develop in recent to the engagement of each TSU for communication and governing the jamming signal based on the spectrum engagement. The proposed approach minimizes the delay due to jamming signaling in existing system. The Experimental results obtained shows an enhancement to the system throughput, fairness factor and minimizes the delay metric for proposed approach.
\end{abstract}

Keywords-Slot monitoring; spectrum sensing; primary user (PU); secondary user (SU); third-party spectrum utilization (TSU)

\section{INTRODUCTION}

The evolution of wireless communication has resulted in the interfacing of various advanced services on portable communication devices, with many new services being integrated at a rapid pace. The incorporation of new services requires a higher data rate and high offers service quality. Wherein users are increasing rapidly, and the services integrated are also increasing, it is the need of the existing wireless communication system to improve the communication architecture to offer the best data exchange. The wireless spectrum constraint has given rise to the concept of spectrum sharing, in which users are interfaced to use the free spectrum of other users to meet the newly evolving service demand. The most suitable approach is the cognitive radio network (CRN) where each user interfaces with other users for spectrum sharing. The advantage of spectrum sharing has resulted in higher service compatibility. However, the issues of spectrum sensing, and allocation are critical in these networks. The sensing of the available spectrum with minimal network overhead is the primary requirement for an efficient CRN operation. In the sharing of free spectrum, primary user (PU) and secondary user (SU) undergoes a communication phase for signal status sensing and based on signal energy spectrum sensing is developed. In improving the objective of spectrum sensing, new methods of recurrent learning approaches, fusion based approach and machine learning techniques were developed. In [1] the accessing data rate is increased by the sensing spectrum of side cognitive nodes which are added into the network with course of time. The sensing operation developed performs spectrum sharing minimizing the packet delivery failure in the network. A multi-scale spectrum sensor in a CRN is outlined in [2]. This method developed a spectrum sensing approach based on an interference-matched design, where more accurate information interfaces with the network for proper communication control. A Spectrum sensing approach in CRN using multi-metric monitoring in an outline in [3]. The spectrum sensing were developed based on the concurrent monitoring of traffic condition, delay and channel usage. In communication system using as frequency division multiplexing [4], spectrum sensing is developed using time-frequency signal estimation. The proposed approach was developed using a blind signal estimation approach, which enhanced the energy detection performance. In [5] for multipath and multi-channel conditions a window-based blind spectrum estimation is proposed. This approach also improved the energy detection performance and hence the spectrum sensing in cognitive radio. An adaptive threshold approach for the detection of spectrum under channel interference is outlined in[6]. This work presented a covariance approach for channel sensing where an adaptive threshold is proposed to reduce the error probability in spectrum sensing. In recent past to improve the blind sensing performance, regression models were used for a faster convergence. A Markov approach for spectrum sensing based on a distributed coordinate function (DCF) is presented in [7]. The approach derives from a Markova chain model in developing a CRN operation for spectrum sensing. In the application of a wide area network, an opportunistic spectrum sensing approach using a cognitive sensor network was presented in [8]. This approach presented a likelihood distribution of channel availability using Markov chain computing for primary users using the spatial distribution of the channel. In other techniques of spectrum sensing advanced 
machine learning approaches were also introduced. In $[9,10]$ a novel fusion model for spectrum sensing is presented. This approach used a machine learning model in which the $\mathrm{k}$ nearest neighbor estimation is used in spectrum sensing. A similar approach for a trust-based spectrum sensing model based on a data fusion model was presented in [11]. The approach developed a 'mechanism design theory' for detecting false requests for spectrum sharing owing to unauthentic users in the network with cognitive nodes. A centralized data fusion model was presented to monitor the node's operation in validating user sensing requests. The author in [12] proposed a multi-channel cooperative spectrum sensing (MCSS) method for optimizing spectrum sensing in a cognitive radio network. This approach develops a novel game theory of node selection to extend energy savings in a cognitive device. In [13], a fusion center (FC) model was proposed for cooperative spectrum sensing. The proposed approach improves the throughput by minimizing the amount of reporting time in the network. The approach is a centralized monitoring scheme of spectrum sensing where the network is divided into two clusters and the spectrum sensing observations are monitored at the FC in deciding for spectrum sensing based on defined fusion rules. Under multi user environment a centralized cooperative spectrum sensing (CSS) approach is presented in [14]. This method was created using k-means shift clustering over local energy vectors. In [15] Formal paraphrase the sensing of opportunistic spectrum by a primary user in the mobility scenario is presented with authentic access to spectrum usage. A k-means approach to spectrum sensing is proposed in which the identification of suspected users in spectrum access is developed for optimal spectrum utilization. In [16] a reinforcement learning (RL) method for cooperative spectrum seeing in CRN operation is presented. This approach developed a channel scanning approach for spectrum sensing and requesting, where a confidence bound approach was proposed for secondary user (SU) spectrum sensing. An approach for a malicious free processing of spectrum allocation in a cognitive radio network was outlined in [17] where the issue of false sensing of the spectrum in the network and allocation is encountered and solved. Similar approach to energy detection for different false alarms and noise variance is outlined in [18]. The presented interface for different communication networks with cognitive devices for sensing the opportunistic spectrum is outlined. In the application of improved spectrum sensing for real time usage a cognitive Internet of Things (CIOT) was presented in [19]. Spatially correlated approach for spectrum sensing using energy-efficient processing in cognitive radio devices. However, to cope with rapid evolving service demands and user accessibility, the spectrum sharing needs a further enhancement, which is overcome by other neighboring cluster node termed as third party users [20]. These users share spectrum to nodes which are not registered into their clusters. To sense such user spectrum, nodes generate a jamming signal for reading the signaling status of the node. In this case, the random jamming signal is generated by different nodes to detect the accessing free spectrum. However, a random jamming signal leads to delay in node operation and intern reduces the network throughput. In [21] a sparse based coding approach for detection of jamming attack is presented. This method proposed a dictionary is used in classifying the spectrum hole and a jamming state. The dictionary is used by machine learning approach in classification of the user emulation. A max-min method for the optimization of energy saving in congestive sensor network is outlined in [22]. The proposed approach schedules the request a node operational unit of the sensing spectrum based on the channel sensing of a node. A Rendezvous algorithm using channel Hopping $(\mathrm{CH})$ for spectrum sensing in asynchronous and asymmetric model is presented in [23]. The jamming conditions were minimized by a Hybrid Rendezvous Algorithm which operates on jamming and non-jamming conditions. The jamming signaling was observed for minimizing the jamming conditions in cognitive network. However, the past developments observe the jamming condition based on a pre existing dictionary model or based on the channel conditions. In these approaches the requesting overhead is not addressed and a repetitive jamming signaling for spectrum sensing leads to a large delay. In this paper a new monitoring approach for user engagement based on channel occupancy is proposed. The centralized fusion monitoring of the channel engagement leads to minimization of requesting overhead for high engaged users, resulting in minimization of jamming delay. The outcomes of the presented work are:

1) A new method for monitoring of jamming signal based on user's engagement is proposed.

2) The Jamming overhead due to random scheduling is minimized.

3) The Delay metric is minimized and throughput is improved by the monitored approach of spectrum engagement.

The rest of this paper is outline in 6 sections. The system outline and the operation of jamming signaling in cognitive radio network for spectrum sensing is outlined in Section 2. Section 3 presents the process of spectrum allocation using jamming condition. The proposed approach of slot monitoring is outlined in Section 4. Experimental result observations for the developed approach are outlined in Section 5. Section 6 presents the conclusion of presented work.

\section{System OutLine}

A cognitive radio network (CRN) consists of a Secondary User (SU) -Tx/Rx, a relay unit, and a Primary User (PU) $\mathrm{Tx} / \mathrm{Rx}$ unit. These communication units are equipped with a single transmitting and receiving antenna. All nodes in this connection are in full-duplex operation. In the communication of data packets, information is exchanged using a relay node. The uplink of a packet is made based on the position of the users in the network. A generic architecture of a CRN system is shown in Fig. 1. 


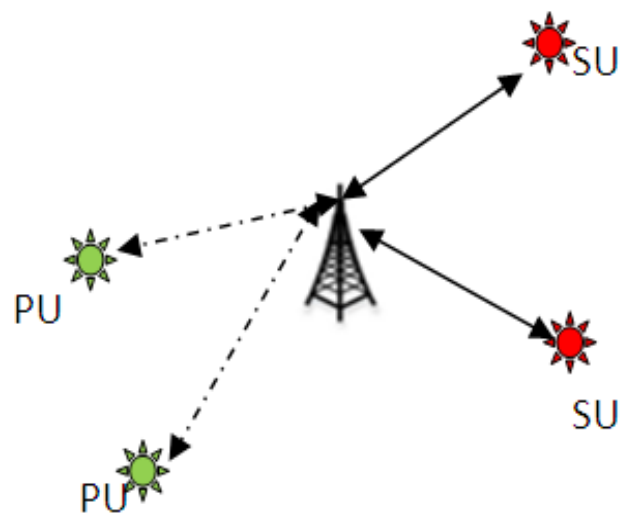

Fig. 1. Communication Architecture of the CRN System.

In the spectrum sensing operation, the Secondary User (SU) detects the freely licensed Primary User (PU) spectrum when the user does not use the spectrum to communicate. Spectrum availability is estimated using an energy detection approach of signal energy. This method uses a threshold-based estimation technique which operates under two hypotheses, $\mathrm{h}_{0}$ and $h_{1}$, respectively,

$$
\begin{aligned}
& h_{0}: X_{i}=N_{i} \\
& h_{i}: X_{i}=S_{i}+N_{i}
\end{aligned}
$$

Hypotheses $h_{0}$ specifies the nonexistence of a signal, where $h_{1}$ denotes the occurrence of the signal and the coupling of the PU spectrum by the transmission signal $S_{i}$ interfering with the additive Gaussian noise of the channel with mean zero and variance $\sigma$. Under the generalized likelihood Gaussian distribution, the probability of estimation for the two hypotheses $\mathrm{h} 0$ and $\mathrm{h} 1$ is defined as,

$$
\begin{aligned}
& E^{\prime}=\frac{1}{k} \sum_{i=1}^{k}\left(\frac{\left|y_{i}\right|}{\sigma}\right)^{m}>t h \quad\left({\text { For hypothesis } \mathrm{h}_{0)}}^{m}\right. \\
& =\frac{1}{k} \sum_{i=1}^{k}\left(\frac{\left|y_{i}\right|}{\sigma}\right)^{m}<\text { th }\left(\text { for hypothesis } \mathrm{h}_{1}\right)
\end{aligned}
$$

Here $m>0$ is a random static value where the threshold is defined by the channel variance. For usage of the additional spectrum from other clusters, a third-party secondary user (TSU) was used. The spectrum sharing operation with TSU operation is illustrated in Fig. 2.

In the detraction of spectrum, a medium access control (MAC) layer approach using jamming signals termed as probing functions is used. The probing function is developed as a trial and error-based approach where a jamming signal is used in the detection of channel occupancy. This process generates a jamming signal and senses the behavior of a user in sensing the occupied channel. As a result, the holding delay for TSU is longer, resulting in a lower throughput. The delay is observed as an overhead on the network due to a jammed signal. The nodes here generate the jamming signal on a random basis by sensing the free spectrum from TSU. The operation of the probing function in the spectrum sensing operation is outlined in Fig. 3.

The random generation of the Jamming signal results in higher overhead and introduces a large delay in the network. This overhead minimizes network throughput. To improve the sensing performance, a slot-monitored jamming system is proposed. This approach records the channel engagement, and the jamming is generated for the node with minimum channel allocated. Here, each node in the network shares the channel engagement with a monitoring central node, and all the requests are processed on the decision of this node. The illustration of the slot sensing approach is shown in Fig. 4.

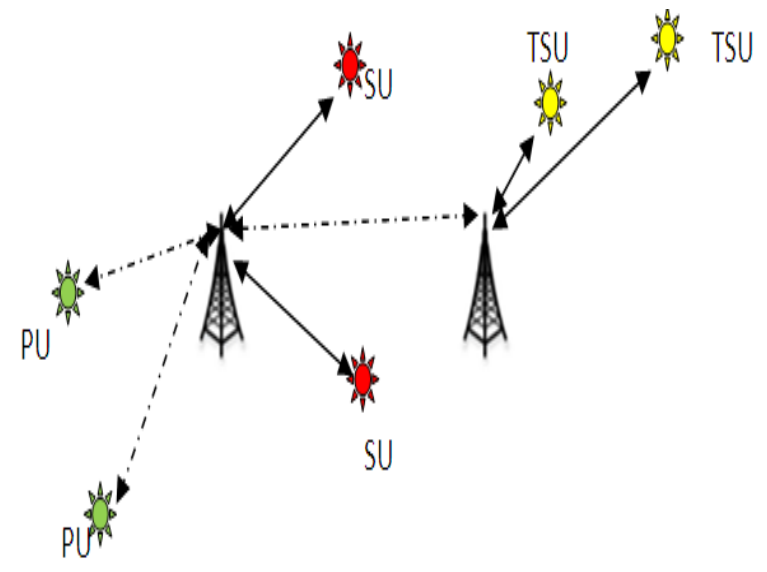

Fig. 2. CRN System with TSU Interface.
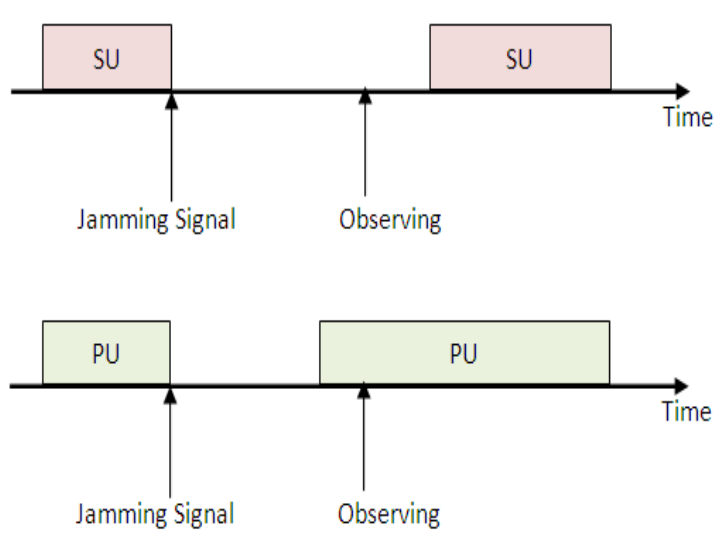

Fig. 3. Probing Function for Spectrum Sensing.

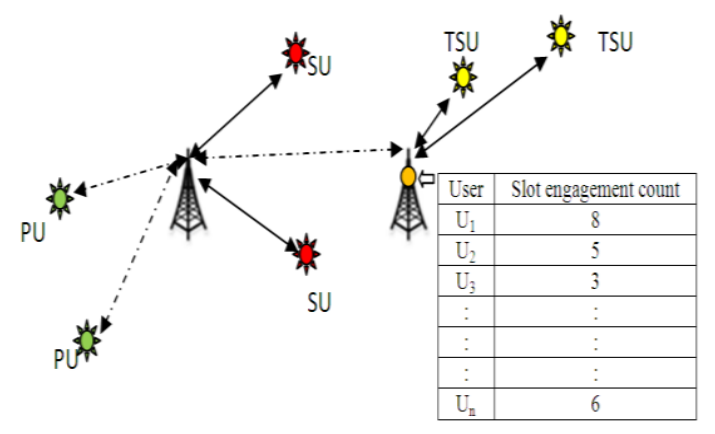

Fig. 4. The Proposed Approach of Slot Monitored Probing. 
The existing approach of random jamming-based spectrum sensing, and the proposed approach of slot monitored spectrum sensing are outlined in the following section.

\section{SPECTRUM UTILIZATION CODING}

The signal estimators are limited by the channel diversity. The signals are estimated with prior knowledge of channel parameters and a recursive process performs the estimation error minimization in recovering the originally transmitted information. The diversity in propagating channels and the variant data rate service demand have led to a minimization of the estimation performance.

The limiting constraint to wireless communication is the convergence period where estimation is expected to be as near the actual transmitted information. In the approach of estimation, the recursion process affects the convergence period, and a larger delay is observed for a diverse channel condition. The estimator is expected to process estimation at a faster rate to minimize the convergence delay.

In the estimation of communicating signal under a channel variant condition, the communicating node is processed for spectrum sensing using an energy detection model. The estimation of the signal under the channel variation plays a critical role in the detection of free spectrum in the network.

In the allocation process, the third-party secondary user defines the spectrum sensing operation as a state of transition illustrated as Fig. 5.

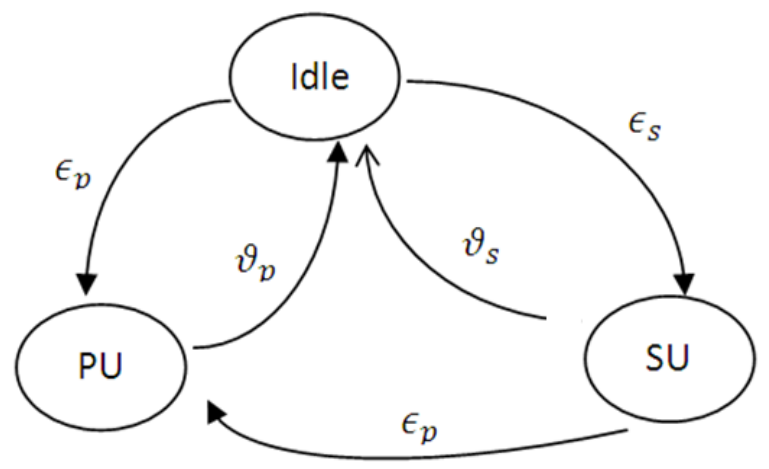

Fig. 5. Transition Diagram for Spectrum Sensing.

In the spectrum sensing process, each of the channels is processed for spectrum sensing, where the node remains in an idle state, indicating free channel availability. The PU and SU nodes represent the used and occupied channels. In spectrum sensing operations, a probing function is defined, which is integrated with a jamming operator to sense the free channel availability. In the process of the sensing operation, the jamming operator passes a value of ' 1 ' when the node has to go for sensing. The jamming probability of a node, in this case, is given by ' $p$ ' where $0<p<1$. The value of ' $P_{\text {min }}$ ' indicates that the winning probability of the node for a SU is obtained by jamming operation. Each time channel access is achieved, a throughput variation is observed. In this case, the throughput gain is changed from, $\pi_{p}$ to $\pi_{p}^{\prime}$ which is given as,
Where,

$$
\pi_{p}^{\prime}=0.99 \times \pi_{p}
$$

Where,

$$
\pi_{p}=\frac{\varepsilon_{p}}{\varepsilon_{p}+v_{p}}
$$

Here, the sensing probability is based on the energy detection and rate of a jamming signal generated. The jamming condition generated in the network introduces a delay for each request generated. This gives a higher probability of delay in the spectrum sensing operation. To avoid the spectrum sensing operation, in this work a new slot allocation approach is proposed. The operation outline of the conventional approach is given, as is the arrival and departure rate of the primary user (PU).

The value of jamming probability is defined by,

$p=1-\left(1-p_{n}\right)^{n}$

Here,

\section{$p_{n}$ Is the jamming rate.}

$\mathrm{n}$ is the spectrum unit size.

In a wireless network, for spectrum sensing, the node generates a jamming signal at a probability of ' $p$ '. The aggregated time for a k-third party user node to allocate for sensing is given by,

$$
\pi_{i}=\sum_{i=1}^{n} N_{i} \times P_{i}
$$

\section{Where}

$\mathrm{N}_{\mathrm{i}}$ is the number of third-party secondary users increasing $P_{i}$ is the probability of jamming associated with each node.

The system is developed using an assumption of known channel variation. The jamming error is taken as a reference for the prediction of signal in estimation. The maximum channel interference is defined as a non-linear time variant interference observed in the channel. The variation in channel parameters leads to an unstable condition of signal estimation and hence leads to improper sensing. To improve the estimation performance, in this study, a new approach to slotbased monitoring of channels is proposed.

\section{SLOt MONITORING Probing FUnCTION FOR JAMMING OPERATION}

For the spectrum sensing operation, each primary user requests the secondary user for a free spectrum using a jamming signal. In the process of the spectrum sensing and allocation of spectrum, the node generates multiple requests for jamming from the secondary node. This repetitive request results in overhead for the secondary node and channel congestion. To avoid the repetitive requests in this study, a 
slot monitoring approach is proposed. In this approach, a monitoring parameter called slot count $\left(s_{n}\right)$ is introduced. This approach monitors all the registered users' slot allocations engaged in communication. In the process of communication, each of the user nodes allocated for communication is allotted a slot to keep updated with a centralized monitoring node at a macro cell level. This node centralizes all registered users and records the allocated slots. In the occupied channel, the aggregated slot count is given by,

$s_{n}=\sum_{i=1}^{n} s_{i}$

indicates the channel slot that is currently occupied. Each of the allocated slots in this case is given a value of '1' when occupied and ' 0 ' when released. The aggregated slot count results from the overall occupied channels in the network. A repository for the allocated slots in the network is made at the centralized node where K TSU nodes are registered. The repository structure was built as.

$R=\left[s_{n 1,} s_{n 2,} s_{n 3, \ldots \ldots \ldots . . .} s_{n k}\right]$

This repository is used as a reference unit for the allocation permission for slot allocation. In the spectrum sensing operation, the primary user (PU) requests the secondary user's spectrum via a centralized monitoring node. For each of the requests generated for a SU or TSU, the centralized node searches for a slot allocation level. In this case, the monitoring unit process is used to select a node for a request that meets the minimal condition.

$$
T S U_{\text {sens }}=\min (R)
$$

This means that the minimal engaged node is selected in the process of spectrum sensing to avoid non-regular jamming of the node.

The aggregated jamming probability for a secondary node is observed as,

$p_{\text {agg }}=\sum_{i=1}^{k} p_{i} \times t_{i}$

Where $p_{i}$ is the jamming rate for a node and $t_{i}$ is the time period allocated for jamming. This delay is minimized by.

$p_{\text {agg }}=\sum_{i=1}^{k} p_{\text {isel }} \times t_{i}$

The overall spectrum sensing contention delay is minimized by the allocation of the selected node jamming rate $p_{\text {isel }}$ for the request made. This proposed approach minimizes the overall overhead of sensing requisition and the jamming probability of a node.
The algorithm for the proposed work is outlined as follows:

\begin{tabular}{|c|c|}
\hline \multicolumn{2}{|c|}{ Algorithm } \\
\hline \multicolumn{2}{|c|}{ Input: Allocated slot $\left(\mathrm{S}_{\mathrm{i}}\right)$, Repository $(\mathrm{R})$} \\
\hline \multicolumn{2}{|c|}{ Output: Jamming Probability } \\
\hline \multicolumn{2}{|c|}{ Do, } \\
\hline & $\begin{array}{l}\text { Step 1: Create Repository }(R) \text { by registering all nodes of } \\
P U, S U \text { and TSU to centralized monitoring node, }\end{array}$ \\
\hline & $R=\left[s_{n 1,} s_{n 2,} s_{n 3, \ldots \ldots \ldots . . .} s_{n k}\right]$ \\
\hline & $\begin{array}{l}\text { Step 2: Allocate ' } 1 \text { ' for a slot engaged and '0' for a free } \\
\text { channel }\end{array}$ \\
\hline & $\begin{array}{l}\text { Step 3: Request jamming from PU to a centralized } \\
\text { node, for TSU }\end{array}$ \\
\hline & $\begin{array}{l}\text { Step 4: compute slot allocation count for request } T S U \text {, } \\
S_{n}=\sum_{i=1}^{n} S_{i}\end{array}$ \\
\hline & $\begin{array}{l}\text { Step 5: allocate jamming on satisfying allocation } \\
\text { criterion. }\end{array}$ \\
\hline & $\begin{array}{l}\text { Step 6: On allocation criterion not satisfied, search best } \\
\text { possible allocation. } T S U_{\text {sens }}=\min (R)\end{array}$ \\
\hline & Step 7: allocate the probable TSU to requesting $P U$ \\
\hline & Step 8: Performing a Jamming probing. \\
\hline & Step 9: Sense the free spectrum \\
\hline & $\begin{array}{l}\text { Step 10: Request and allocate the TSU spectrum. } \\
\text { Step 11: Update the allocation status to Repository }(R) \text {. }\end{array}$ \\
\hline End & \\
\hline
\end{tabular}

\section{RESUlT AND DiSCUSSION}

The proposed approach is evaluated for a cognitive network with random users deployed under channel variation conditions. The approach can update the error weight value based on the jamming operation. The basic design model of a jamming estimator is defined by a state updating process where a Jamming error response is made in the responsibility of jamming error. A set of the processing bits is shown in Fig. 6.

The request developed by the TSU node for sensing the free spectrum is received at the receiver. The jamming requests are generated for a requesting node to detect the node spectrum availability. Fig. 7 shows the jamming request received at the receiver node.

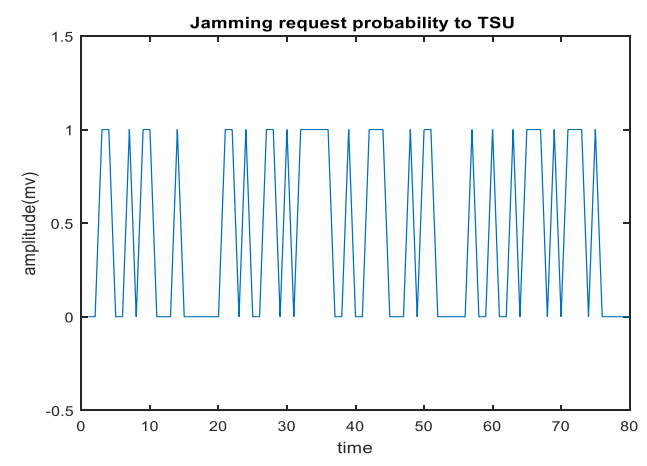

Fig. 6. Jamming Request Probability to TSU. 


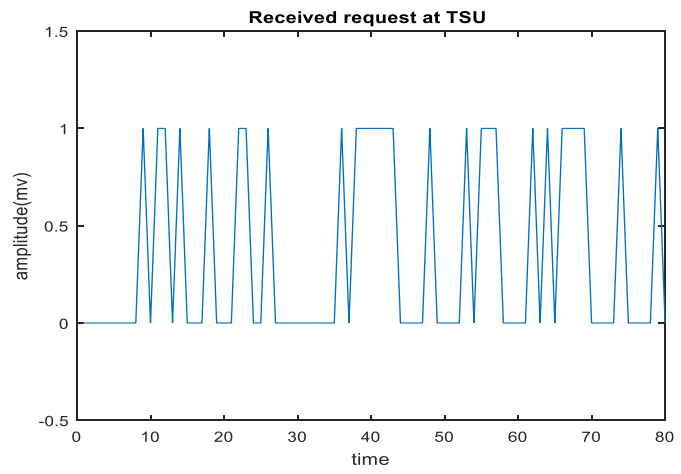

Fig. 7. Received Request at TSU.

In the detection of spectrum availability from a third-party secondary user (TSU), a copy of the received signal is measured at the receiver as illustrated in Fig. 8. The received signal is used for energy detection in the spectrum sensing approach.

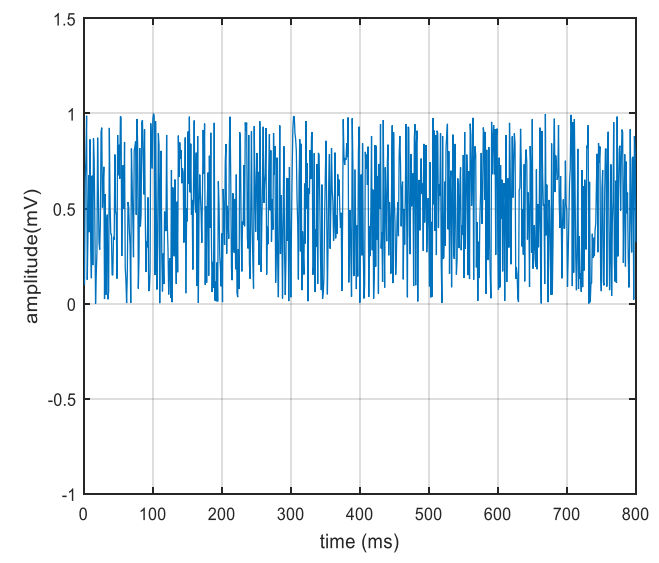

Fig. 8. Signal Received from the SU.

For this affected signal the channel estimation logic is applied. The Energy detection level for the communicating signal at the receiver is illustrated in Fig. 9.

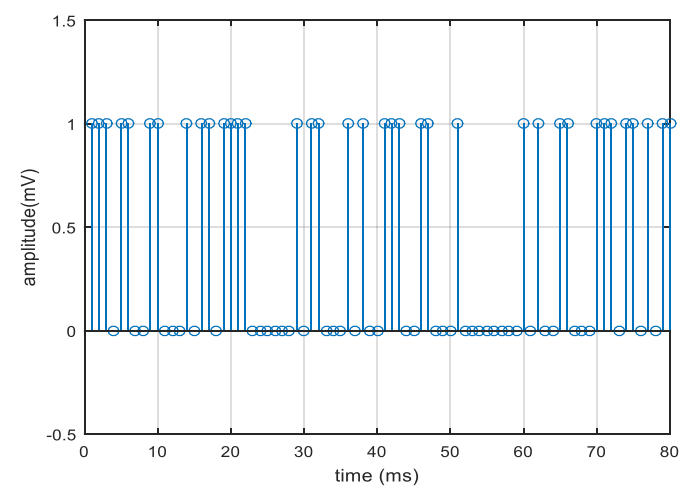

Fig. 9. Energy Detection Level at PU.

The availability of the additional spectrum by the TSU unit results in faster packet delivery. However, the spectrum sensing is made based on the offered jamming rate to the TSU unit. The overhead per node due to variation in jamming rate is presented in Fig. 10. The observation illustrates a lower overhead by the proposed slot monitoring probing approach compared to the existing with and without probing function. A lower overhead lead to higher throughput in the network. With an increase in jamming rate, the overhead is controlled to a minimum by slot monitored probing compared to with and without probing.

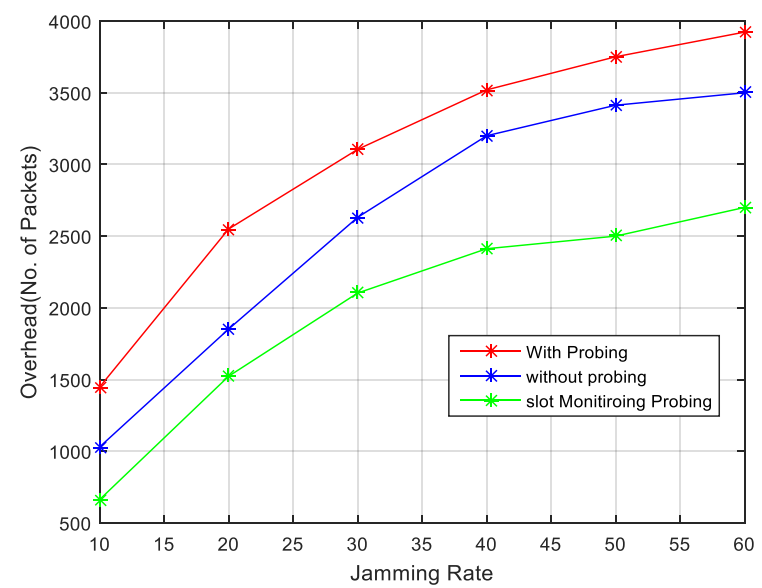

Fig. 10. Overhead for Varying Jamming Condition.

Observations of the overhead due to varying jamming rates and streaming sizes for the developed approaches are listed in Tables I and II, respectively.

In the streaming of signals, the signals are propagated over an $\mathrm{m} \times \mathrm{n}$ channel. The channel diversity results in dynamic distortion in communicating signals, leading to higher errors. The observed fairness to the communicating signal defined with respect to signal error is illustrated in Fig. 11.

TABLE I. OVERHEAD (\%) FOR CONTROL STREAMS WITH VARYING JAMMINGRATE

\begin{tabular}{|l|l|l|l|}
\hline $\begin{array}{l}\text { Jamming Rate } \\
(\mathbf{p})\end{array}$ & With probing & $\begin{array}{l}\text { Without } \\
\text { probing }\end{array}$ & $\begin{array}{l}\text { Slot monitored } \\
\text { probing }\end{array}$ \\
\hline 10 & 1448 & 1030 & 663 \\
\hline 20 & 2550 & 1852 & 1523 \\
\hline 30 & 3105 & 2631 & 2103 \\
\hline 40 & 3520 & 3200 & 2412 \\
\hline 50 & 3751 & 3413 & 2500 \\
\hline 60 & 3923 & 3500 & 2700 \\
\hline
\end{tabular}

TABLE II. OVERHEAD (\%) EVALUATION WITH RESPECT TO CONTROL STREAMS WITH VARYING STREAM SIZE

\begin{tabular}{|l|l|l|l|}
\hline Stream size & With probing & $\begin{array}{l}\text { Without } \\
\text { probing }\end{array}$ & $\begin{array}{l}\text { Slot monitored } \\
\text { probing }\end{array}$ \\
\hline 500 & 106.423 & 93.926 & 92.32 \\
\hline 600 & 309.64 & 250.65 & 152.14 \\
\hline 700 & 500.32 & 350.98 & 215.69 \\
\hline 800 & 645.293 & 440.00 & 250.14 \\
\hline 900 & 735.853 & 555.48 & 333.45 \\
\hline 1000 & 887.823 & 748.12 & 447.89 \\
\hline
\end{tabular}




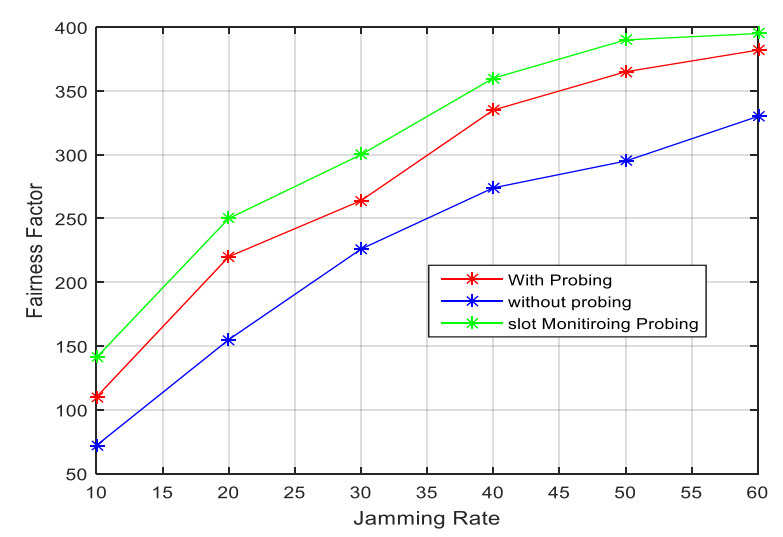

Fig. 11. Fairness Factor over Varying Jamming Rate.

TABLE III. FAIRNESS FACTOR WITH VARYING JAMMING RATE

\begin{tabular}{|l|l|l|l|}
\hline $\begin{array}{l}\text { Jamming Rate } \\
(\mathbf{p})\end{array}$ & With probing & $\begin{array}{l}\text { Without } \\
\text { probing }\end{array}$ & $\begin{array}{l}\text { Slot monitored } \\
\text { probing }\end{array}$ \\
\hline 10 & 72 & 110 & 141 \\
\hline 20 & 155 & 220 & 250 \\
\hline 30 & 226 & 264 & 300 \\
\hline 40 & 274 & 335 & 360 \\
\hline 50 & 295 & 365 & 390 \\
\hline 60 & 330 & 382 & 395 \\
\hline
\end{tabular}

TABLE IV. FAIRNESS FACTOR WITH VARYING STREAM SIZE

\begin{tabular}{|l|l|l|l|}
\hline Stream size & $\begin{array}{l}\text { With } \\
\text { probing }\end{array}$ & Without probing & $\begin{array}{l}\text { Slot monitored } \\
\text { probing }\end{array}$ \\
\hline 500 & 92 & 100 & 110 \\
\hline 600 & 150 & 260 & 315 \\
\hline 700 & 160 & 380 & 520 \\
\hline 800 & 180 & 480 & 660 \\
\hline 900 & 200 & 600 & 750 \\
\hline 1000 & 220 & 800 & 890 \\
\hline
\end{tabular}

The lower overhead in the proposed approach offers a higher communicating throughput when compared to with and without probing. The throughput increased due to slot monitoring is presented in Fig. 12. The throughput is observed to be equal at the lower jamming rate. However, with an increase in the jamming rate, the proposed approach outperforms the existing approach due to proper slot monitoring.

The diversity in the channel model results in higher interference impact which introduces distortion to the signal in a dynamic manner. The estimator units observe different convergence times for the estimation of signal in such a case. The time taken builds the overall communication delay, which is presented in Fig. 13. The delay for the proposed approach is observed to be reduced as compared to the existing approach.

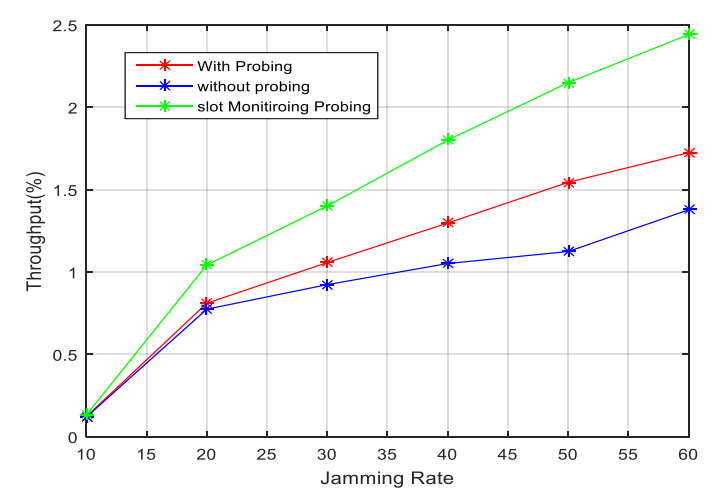

Fig. 12. Throughput over Jamming Rate for the Developed Approaches.

TABLE V. Throughrut Gain (\%) WITH a VARYing JAMming RATE

\begin{tabular}{|l|l|l|l|}
\hline $\begin{array}{l}\text { Jamming Rate } \\
(\mathbf{p})\end{array}$ & With probing & $\begin{array}{l}\text { Without } \\
\text { probing }\end{array}$ & $\begin{array}{l}\text { Slot monitored } \\
\text { probing }\end{array}$ \\
\hline 10 & 0.120 & 0.123 & 0.133 \\
\hline 20 & 0.775 & 0.811 & 1.043 \\
\hline 30 & 0.924 & 1.058 & 1.402 \\
\hline 40 & 1.052 & 1.298 & 1.801 \\
\hline 50 & 1.125 & 1.545 & 2.150 \\
\hline 60 & 1.378 & 1.725 & 2.442 \\
\hline
\end{tabular}

TABLE VI. Throughrut Gain (\%) with a VAR Ying Stream Size

\begin{tabular}{|l|l|l|l|}
\hline $\begin{array}{l}\text { Stream } \\
\text { size }\end{array}$ & With probing & Without probing & $\begin{array}{l}\text { Slot monitored } \\
\text { probing }\end{array}$ \\
\hline 500 & 0.500 & 0.520 & 0.536 \\
\hline 600 & 0.599 & 0.616 & 0.845 \\
\hline 700 & 0.725 & 1.025 & 1.425 \\
\hline 800 & 1.018 & 1.354 & 2.056 \\
\hline 900 & 1.858 & 2.256 & 2.547 \\
\hline 1000 & 2.568 & 2.956 & 3.452 \\
\hline
\end{tabular}

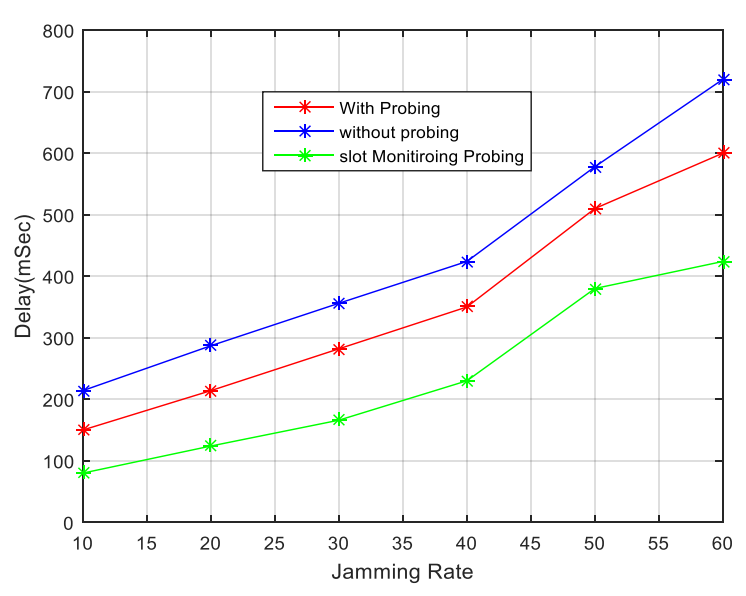

Fig. 13. Convergence Time Delay (msec) Performance for the Developed Approaches. 
TABLE VII. DELAY Timing (MSEC) WITH VARYING JAMMING RATE

\begin{tabular}{|l|l|l|l|}
\hline $\begin{array}{l}\text { Jamming Rate } \\
(\mathbf{p})\end{array}$ & With probing & $\begin{array}{l}\text { Without } \\
\text { probing }\end{array}$ & $\begin{array}{l}\text { Slot monitored } \\
\text { probing }\end{array}$ \\
\hline 10 & 214 & 150 & 80 \\
\hline 20 & 287 & 214 & 124 \\
\hline 30 & 356 & 282 & 166 \\
\hline 40 & 424 & 350 & 230 \\
\hline 50 & 578 & 510 & 380 \\
\hline 60 & 720 & 600 & 424 \\
\hline
\end{tabular}

TABLE VIII. Delay Timing (MSEC) With VARying STREAm Size

\begin{tabular}{|l|l|l|l|}
\hline Stream size & With probing & $\begin{array}{l}\text { Without } \\
\text { probing }\end{array}$ & $\begin{array}{l}\text { Slot monitored } \\
\text { probing }\end{array}$ \\
\hline 500 & 261 & 202 & 35 \\
\hline 600 & 350 & 288 & 55 \\
\hline 700 & 434 & 380 & 74 \\
\hline 800 & 517 & 472 & 103 \\
\hline 900 & 705 & 688 & 170 \\
\hline 1000 & 879 & 809 & 190 \\
\hline
\end{tabular}

\section{CONCLUSION}

In the process of service provisioning in a heterogeneous network, the prime issues observed are the processing overhead due to multiple network interfacing. The CRN can provide higher network connectivity; however, the CRN is limited by the device capability. Towards improving the performance of CRN operations, a third-party spectrum sensing approach has been developed. Their spectrum sensing and usage by a neighboring cell result in third-party users, which are sensed for free spectrum as an additional resource for wireless communication. The proposed approach of slot monitoring probing results in higher network throughput and reduced delay parameters. The proposed approach of slot monitoring probing results in a minimization of overhead by 1000-1500 packets with the increase in jamming rate. The fairness factor is improved to 90 packet accuracies compared to the existing approach. The throughput improved by $1.5 \%$ compared to the existing approach. The proposed approach obtains a minimization of $200 \mathrm{msec}$ delay. The approach developed the utilization of secondary cellular spectrum utilization for faster transmission.

\section{REFERENCES}

[1] A. Jaya Lakshmi, G. N. Swamy, M. N. Giriprasad, "Optimum channel allocation for QOS Provisioning in Cognitive Multihop radio adhoc networks,"International Journal of Intelligent Engineering and Systems, Vol. 11,February 2018, pp 137-146.

[2] N. Michelusi, M. Nokleby, U. Mitra and R. Calderbank, "Multi-Scale Spectrum Sensing in Dense Multi-Cell Cognitive Networks," IEEE Transactions on Communications, Vol. 67, No. 4, pp. 2673-2688, April2019.

[3] D.Ramesh and N. Venkatram, "Current State of Benchmarking Spectrum Sensing and Routing Strategies in Cognitive Radio Ad Hoc Networks," Journal of Theoretical \& Applied Information Technology, Vol.96, 2018, pp. 3490-3510.

[4] Q. Cheng, Z. Shi, D. N. Nguyen and E. Dutkiewicz, "Deep learning network based spectrum sensing methods for OFDM systems" in arXiv:
1807.09414, 2019.

[5] J. Vartiainen, H. Karvonen, M. Matinmikko-Blue, L. Mendes, H.Saarnisaari, and A. Matos, "Energy Detection Based Spectrum Sensing for Rural Area Networks," EAI Endorsed Transactions on Wireless Spectrum, Vol. 4, April 2020.

[6] C. Charan and R. Pandey, "Intelligent selection of threshold in covariance-based spectrum sensing for cognitive radio networks," Wireless Networks, Vol. 24, November 2018, pp.: 3267-3279.

[7] J. Peng, "Throughput analysis of the IEEE 802.11 DCF in cognitive radio networks," Procedia Computer Science, Vol.151, January 2019, pp 264-271.

[8] T. V. Saroja, L. L. Ragha, and Satyendra Kumar Sharma, "A Dynamic Spectrum Access Optimization Model for Cognitive Radio Wireless Sensor Network," ICTACT Journal on Communication Technology, Vol.8, September 2017, pp.1559-1565.

[9] H. A. Shah and I. Koo, "Reliable machine learning based spectrum sensing in cognitive radio networks", Wireless Communication Mobile Computing, vol. 2018, September 2018.

[10] H. B. Ahmad, "Ensemble classifier based spectrum sensing in cognitive radio networks," Wireless Communications and Mobile Computing, vol. January 2019, Article ID 9250562, 16 pages.

[11] J.Wang, I. Chen, Jeffrey J. Tsai, and D.Wang,"Trust-based mechanism design for cooperative spectrum sensing in cognitive radio networks," Computer Communications, Vol.116, 2018, pp.90-100.

[12] A. Bagheri, A. Ebrahimzadeh, and M.Najimi, "Game-theory-based lifetime maximization of multi-channel cooperative spectrum sensing in wireless sensor networks," Wireless Networks, Vol.26, May 2020, pp. 4705-4721.

[13] M. V. S. Sairam and M. Sivaparvathi, "Reduction of Reporting Time for Throughput Enhancement in Cooperative Spectrum Sensing Based Cognitive Radio," International Journal of Intelligent Engineering and Systems, Vol.11, February 2018, pp. 163-170.

[14] S. Zhang, Y. Wang, P. Wan, J. Zhuang, Y. Zhang and Y. Li, "Clustering Algorithm-Based Data Fusion Scheme for Robust Cooperative Spectrum Sensing," IEEE Access, Vol. 8,January 2020, pp. 5777-5786.

[15] S. Bayhan, A. Zubow, P. Gawłowicz and A. Wolisz, "Smart Contracts for Spectrum Sensing as a Service," IEEE Transactions on Cognitive Communications and Networking, Vol. 5, September 2019, pp. 648-660.

[16] W. Ning, X. Huang, K. Yang, F. Wu, and S. Leng ,"Reinforcement learning enabled cooperative spectrum sensing in cognitive radio networks," Journal of Communications and Networks, Vol.22, February 2020, pp. 12-22.

[17] X. Luo, "Secure Cooperative Spectrum Sensing Strategy Based on Reputation Mechanism for Cognitive Wireless Sensor Networks." IEEE Access, Vol.8, July 2020, pp.131361-131369.

[18] M. A. Saad, S. T. Mustafa, M. Hussein Ali, M. M. Hashim, M. B. Ismail, and A. H. Ali, "Spectrum sensing and energy detection in cognitive networks," Indonesian Journal of Electrical Engineering and Computer Science, Vol.17, June 2020, No. 1, pp.465-472.

[19] R. Wan, M. Wu, L. Hu and H. Wang, "Energy-Efficient Cooperative Spectrum Sensing Scheme Based on Spatial Correlation for Cognitive Internet of Things", IEEE Access, Vol. 8,July 2020, pp. 139501-139511.

[20] R. Ganesh Babu , Mohammad S. Obaidat, and Rajesh Manoharan, "Comparative analysis of distributive linear and nonlinear optimized spectrum sensing clustering techniques in cognitive radio network systems." IET Networks, Vol .10, May 2020,pp.253-263.

[21] H. M. Furqan, M. A. Aygul, M. Nazzal, H.Arslan. "Primary user emulation and jamming attack detection in cognitive radio via sparse coding." EURASIP Journal on Wireless Communications and Networking, Vol-2020, No.1, 2020, pp-1-19.

[22] Zhang, Z. Jian Chen, Lu Lv, Q. Ye "Utilizing Cooperative Jamming to Secure Cognitive Radio NOMA Networks." In GLOBECOM 2020-2020 IEEE Global Communications Conference, pp. 1-6. IEEE, 2020.

[23] A. Gabriel, Y. Kim. "Hybrid Rendezvous Algorithm against Jamming Attacks for Cognitive Radio Networks."Journal of Communication, Vol.13, No.4, 2018, pp-169-174. 